\title{
MATHEMATICAL MODELS FOR FLAW AND DAMAGE TOLERANCE ANALYSES
}

\author{
BARNA Szabó \\ Washington University \\ St. Louis, MO 63130 USA \\ szabo@wustl.edu
}

[Received: August 11, 2014, Accepted: September 4, 2014]

Dedicated to István Páczelt on the occasion of his 75th birthday.

\begin{abstract}
This paper is concerned with the conceptual development of mathematical models suitable for reliable prediction of fatigue life in metallic mechanical and structural components. Of particular interest is the prediction of the number of loading cycles to failure in parts that contain irregularities in material properties, inclusions and surface features caused by corrosion or other damage and are subjected to periodic loading.
\end{abstract}

Mathematical Subject Classification: 65N30, $65 \mathrm{Z} 05$

Keywords: Validation, metal fatigue, fracture mechanics, mathematical model

\section{INTRODUCTION}

Conceptual development of mathematical models is an inductive process that involves expert opinion, virtual experimentation and calibration. The end product of conceptualization is a mathematical model. This paper is concerned with aspects of conceptual development of mathematical models designed to support condition-based maintenance (CBM) and reliability-centered maintenance (RCM) decisions. The intended use of such models are: (a) identification of maintenance problems early, when they can be corrected at a relatively low cost and (b) scheduling maintenance only when needed, thereby realizing increased asset utilization, extension of equipment life, and reduction in operating costs.

Both CBM and RCM require mathematical models capable of predicting the remaining useful life of parts with a reasonably high degree of confidence. There are two seemingly competing approaches to fatigue life management: Flaw tolerance and damage tolerance. The purpose of flaw tolerance analysis is to evaluate the likelihood of crack initiation from flaws, given some cyclic loading. The purpose of damage tolerance analysis is to estimate crack growth caused by cyclic loading. A comprehensive overview of maintenance practices relating to military aircraft is presented in [1].

The conceptual development outlined in this paper is based on the idea that models formulated for the prediction of crack nucleation, i.e., for the purpose of supporting 
flaw tolerance analysis, and models formulated for the prediction of crack growth, i.e., for the purpose of supporting damage tolerance analysis, are similar in the following sense: In both cases the physical events of interest; crack nucleation and crack growth, are highly nonlinear processes that occur on length scales that are typically less than about $0.5 \mathrm{~mm}$ for aluminum alloys, titanium and steel. The sites of crack nucleation and crack tip regions are called process zones (see, for example [2] and references cited therein). Within the process zone the usual assumptions of infinitesimal strain and small deformation do not hold. Nevertheless, as long as a process zone is completely surrounded by material for which those assumptions hold, crack nucleation and crack extension events occurring within the process zone should be predictable from the solutions of mathematical models that incorporate the assumptions of infinitesimal strain and small deformation only.

The paper is organized as follows: The classical approaches used for the prediction of the effects of notches on the endurance limit and their relationship to linear elastic fracture mechanics are summarized in Section 2 Procedures for the formulation and testing of mathematical models for the prediction of fatigue damage accumulation are outlined in Section 3 Experimental data obtained for twelve micro-machined test specimens made of AF1410 steel are presented in Section 4. Recommendations for a new family of models that account for size effects and removes difficulties associated with the application of linear elastic fracture mechanics in three dimensions are presented in Section 5. A brief summary is presented in Section 6.

\section{Classical models for damage accumulation}

Failure initiation and crack propagation are inherently nonlinear processes that occur on length scales over which the assumptions of infinitesimal strain and small deformation do not hold. Nevertheless, computations based on mathematical models that incorporate the usual infinitesimal strain and small deformation assumptions, coupled with experimentation, have been proven to be useful for predicting crack initiation events and crack propagation rates.

Mathematical models constructed for the prediction of damage accumulation caused by cyclic loading are based on the following assumptions:

1. There exist one or more functionals, computable from the solution of mathematical models based on infinitesimal strain, small deformation theory, that can be correlated with crack initiation events and crack propagation rates with sufficient accuracy to suit the purposes of engineering decision-making.

2. There exist one or more procedures suitable for the generalization of the results of fatigue experiments performed under a particular cyclic loading, characterized by a mean value and constant amplitude, to cyclic loading characterized by arbitrary mean value and constant amplitude.

3. There exist one or more procedures suitable for correlating damage accumulation with variable amplitude cyclic loading, such as loading that represents flight spectra. 
This paper is concerned with the formulation of functionals pertaining to assumption 1. The functionals are called driving forces for damage accumulation or simply driving forces.

2.1. Notch sensitivity. Several approaches have been proposed for the quantification of the effect of notches on the fatigue limit of machine components made of various metallic alloys. Essentially these approaches distinguish between the geometric stress concentration factor $K_{t}$ and the effective stress concentration factor $K_{e}$ where $0<K_{e} \leq K_{t}$.

Definition 1. Nominal stress, defined for notched machine elements subjected to tension, bending and torsion, is understood in machine design to be the maximum normal or shearing stress at a notch computed by formulas based on the assumption that the strain distribution over the cross section is a linear function. Because this definition cannot be generalized to arbitrary domains, unless stated otherwise, we will understand nominal stress to mean the stress that would exist at the location of a notch if the notch were not present.

Definition 2. The geometric stress concentration factor, denoted by $K_{t}$, is the ratio of maximum stress to the nominal stress.

Definition 3. The notch sensitivity index $(q)$ is defined as follows:

$$
q=\frac{K_{e}-1}{K_{t}-1} .
$$

It is dependent on the notch radius $\varrho$ and a material property. Peterson [3], 4] defined the notch sensitivity index as:

$$
q=\frac{1}{1+\alpha / \varrho}
$$

where $\alpha$ is an experimentally determined material constant. Peterson gave approximate values for $\alpha$ for steels as a function of their ultimate tensile strength (UTS). For UTS ranging between 345 and $1725 \mathrm{MPa}$ the estimated range of $\alpha$ is 380 to 33 $\mu \mathrm{m}$ respectively. Although not stated explicitly, there has to be a lower bound on $\varrho$. The experimentally determined values of $q$ for aluminum and steel published in 3 . indicate that $\varrho$ is greater than approximately $\alpha / 4$.

An alternative definition of $q$, based on Neuber's work [5] is:

$$
\bar{q}=\frac{1}{1+\sqrt{\varrho^{\prime} / \varrho}}
$$

where $\varrho^{\prime}$ is an experimentally determined material constant (in length units). It is correlated with UTS in [6]: For UTS ranging between 345 and $1725 \mathrm{MPa}$ the estimated range of $\varrho^{\prime}$ is 430 to $0.9 \mu \mathrm{m}$ respectively.

Investigating notched machine elements subjected to tension, bending and torsion, Neuber formulated the following hypothesis: The driving force for the accumulation of fatigue damage is the elastic stress (shearing and tensile) at a notch tip averaged over a 
material-dependent small distance that has to be determined through experimentation [5].

Neuber's hypothesis and variants of his hypothesis proposed by other investigators form the basis for flaw tolerance analysis. See, for example [8], a reference that makes comparisons among the Neuber-Kuhn, Peterson, Heywood, Stieler-Siebel and the Buch-Switek formulas, all proposed for the prediction of the effects of fillets, characterized by a radius, and notches, characterized by a depth, a notch angle and radius, on the fatigue limit.

Remark 1. Neuber's work was concerned with the fatigue strength of notched machine elements. Averaging stresses over a material-dependent distance for notched bars, shafts and beams can be understood also as averaging over an area or volume. Therefore Neuber's conceptualization admits alternative interpretations on general domains.

2.2. Linear elastic fracture mechanics. Linear elastic fracture mechanics (LEFM) is based on the hypothesis that the driving force for crack propagation under cyclic loading is the amplitude of the stress intensity factor.

Neuber's hypothesis and LEFM may appear to be fundamentally different models of damage accumulation. In reality LEFM can be viewed as a special case of Neuber's model in the following sense: Let us assume that a material-dependent critical distance $d$ exists which is independent of the geometric features or the magnitude of stress or strain. Considering a two-dimensional domain with a crack, assuming that the crack is along the $x$-axis, periodic loading of amplitude $\Delta T_{y}$ is applied in the direction of the $y$-axis and the origin of the coordinate system is the crack tip, the driving force is the average stress $\Delta \sigma_{d}$ induced by $\Delta T_{y}$. By definition;

$$
\Delta \sigma_{d}=\frac{1}{d} \int_{0}^{d} \Delta \sigma_{y}(x, 0) d x .
$$

The periodic loading $\Delta T_{y}$ induces variations in the stress intensity factor ranging between a minimum value $\left(K_{I}\right)_{\min }$ and a maximum value $\left(K_{I}\right)_{\max }$ where $\left(K_{I}\right)_{\min } \geq 0$. Let $\Delta K_{I} \equiv\left(K_{I}\right)_{\max }-\left(K_{I}\right)_{\min }$ and denote by $\left(\Delta K_{I}\right)_{\text {th }}$ the threshold value of $\Delta K_{I}$ below which the crack will not grow. Therefore a crack will not propagate when the amplitude of the average stress is

$$
\Delta \sigma_{d} \leq \frac{1}{d} \int_{0}^{d} \frac{\Delta K_{I}}{\sqrt{2 \pi x}} d x=\Delta K_{I} \sqrt{\frac{2}{\pi d}} .
$$

Equating $\Delta \sigma_{d}$ to the endurance limit $\Delta \sigma_{0}$, we have the following estimate for the critical distance:

$$
d=\frac{2}{\pi}\left(\frac{\left(\Delta K_{I}\right)_{\mathrm{th}}}{\Delta \sigma_{0}}\right)^{2} .
$$

If $\left(\Delta K_{I}\right)$ th and $\Delta \sigma_{0}$ are material properties then $d$ is a material property also. One has to bear in mind however that the endurance limit varies with the size of specimens: When the size increases, the endurance limit decreases. Therefore $d$ is a material 
property only if $\left(\Delta K_{I}\right)_{\text {th }}$ varies with size in the same way as $\Delta \sigma_{0}$. However, for reasons discussed in the following, it is not possible to determine $\left(\Delta K_{I}\right)_{\text {th }}$ accurately.

Example 1. The threshold stress intensity factor for conventionally processed AF1410 steel is approximately $16.0 \mathrm{MPa} \mathrm{m}^{1 / 2}$ and its endurance limit is approximately 950 MPa. Therefore from eq. $(2.6)$ we have $(d=180 \mu \mathrm{m})$. This is an ultra high strength steel; its UTS is approximately $1670 \mathrm{MPa}$. This estimate of $d$ is much larger than the estimate given in [3] and [6]. Note: The ASTM grain size number ${ }^{1}$ of conventionally heat treated AF1410 steel is approximately 10.8 [7]. This corresponds to an average grain size of approximately $8.5 \mu \mathrm{m}$.

It is not difficult to show that the stress intensity factor is proportional to $\sigma_{d} \sqrt{d}$ :

$$
\sigma_{y}(x, 0)=\frac{K_{I}}{\sqrt{2 \pi x}} \rightarrow K_{I}=\sigma_{d} \sqrt{\frac{\pi d}{2}} .
$$

There are two important advantages of using eq. 2.4 instead of eq. 2.7):

1. Equation (2.4) is defined for both cracks and notches whereas eq. (2.7) is defined for cracks only.

2. Problems arise when attempting to interpret the results of physical experiments. Calibration involves correlation of crack growth with load cycles using the assumption that the stress distribution in the test articles very nearly satisfy the conditions of planar elasticity. However the asymptotic expansion of stresses in the neighborhood of a crack tip in two-dimensions is not applicable in three dimensions. This is because asymptotic expansion has a different character in the vicinity of the points where the crack front intersects a free surface. When the specimen is thin then these points are in close proximity and therefore tend to dominate the stress field ahead of the crack tip. When the specimen is thick then the stress fields in the vicinity of these points are usually ignored and the assumption is made that plane strain conditions exist. In reality generalized plane strain conditions exist 9 .

In practical situations, such as shown in Fig. 1 where a crack represented by arc $\mathrm{AB}$ is originating at a countersunk fastener hole, there is substantial epistemic uncertainty as to how crack growth is influenced by the stress field in the vicinity of points A and B. Therefore it is not possible to justify application of conventional LEFM methodology in such cases.

2.3. The theory of critical distances. The classical approaches proposed by Neuber, Peterson and others were precursors to what is called today the theory critical distances (TCD), see [10] - [13]. The line method of theory of critical distances states that for notched components in tension or bending the driving force is:

$$
G_{\mathrm{TCD}}=\frac{1}{2 L} \int_{0}^{2 L} \sigma_{1}(s) d s
$$

\footnotetext{
${ }^{1}$ ASTM Standard E 112 - 96 (2004).
} 


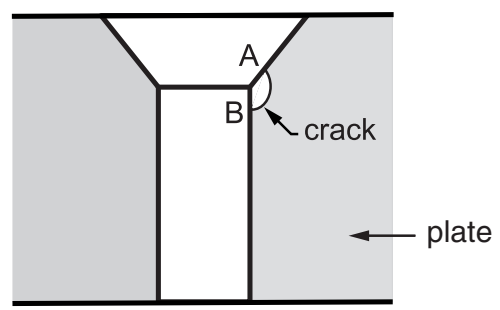

Figure 1. Crack originating at a countersunk hole.

where $L$ is the critical distance, assumed to be a material property, $\sigma_{1}>0$ is the first principal stress, and the domain of integration is chosen such that $G_{\mathrm{TCD}}$ is maximal.

Remark 2. The relationship between the critical distance $d$ defined in eq. 2.6) and the critical distance $L$ defined in eq. 2.8 is $L=d / 2$. See for example [10].

In the following example it is shown that Peterson's definition of $K_{e}$ can be understood as an approximation to the ratio of the average normal stress, in the direction of loading, over the distance $\alpha$, to the nominal stress.

Example 2. We refer to the problem of a circular hole in an infinite plate subjected to unidirectional tension. The notation is shown in Fig. 2. The classical solution for $\sigma_{x}$ is:

$$
\sigma_{x}=\sigma_{\infty}\left[1-\frac{a^{2}}{r^{2}}\left(\frac{3}{2} \cos 2 \theta+\cos 4 \theta\right)+\frac{3}{2} \frac{a^{4}}{r^{4}} \cos 4 \theta\right]
$$

where $\sigma_{\infty}$ is the nominal stress. The geometric stress concentration factor is $K_{t}=$ $\sigma_{\max } / \sigma_{\infty}=3$ where $\sigma_{\max }=\sigma_{x}(a, \pm \pi / 2)$. See, for example, [14].
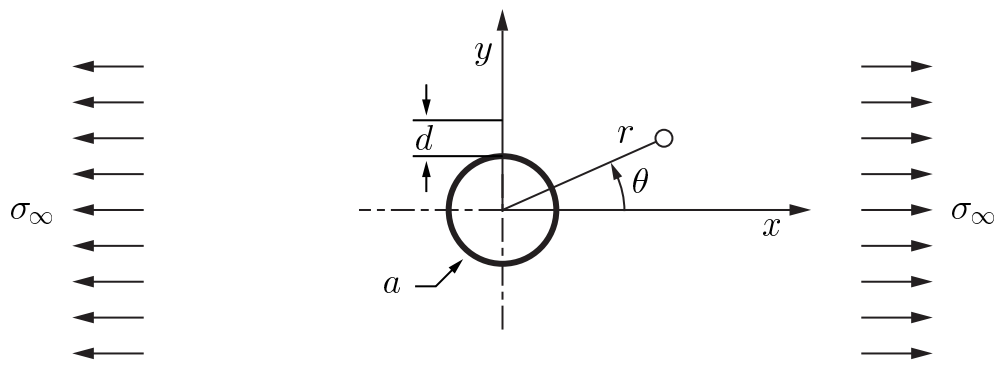

Figure 2. Circular hole in an infinite plate subjected to unidirectional tension $\left(\sigma_{\infty}\right)$. Notation. 
We will be interested in the value of $\sigma_{x}$ averaged over the interval $a \leq y \leq d$. The average value will be denoted by $\sigma_{x}^{(d)}$. Referring to eq. 2.9 , we have

$$
\begin{aligned}
\sigma_{x}^{(d)} & =\frac{1}{d} \int_{a}^{a+d} \sigma_{x}(r, \pi / 2) d r=\frac{\sigma_{\infty}}{d} \int_{a}^{a+d}\left(1+\frac{1}{2} \frac{a^{2}}{r^{2}}+\frac{3}{2} \frac{a^{4}}{r^{4}}\right) d r \\
& =\sigma_{\infty}\left(1+\frac{1}{2} \frac{1}{1+d / a}+\frac{3}{2} \frac{1+d / a+d^{2} /\left(3 a^{2}\right)}{(1+d / a)^{3}}\right) \\
& =\sigma_{\infty}\left(1+2 \frac{1}{1+d / a}-\frac{3}{2} \frac{d / a+2 d^{2} /\left(3 a^{2}\right)}{(1+d / a)^{3}}\right) \\
& =\sigma_{\infty}\left(1+2 \frac{1}{1+d / a}+O(d / a)\right) .
\end{aligned}
$$

Letting $\alpha=d, \varrho=a$ in eq. (2.2) and using eq. 2.1) we have:

$$
K_{e}=\frac{\sigma_{x}^{(d)}}{\sigma_{\infty}}=q\left(K_{t}-1\right)+1
$$

which is the same as eq. 2.10 if we neglect the term of $O(d / a)$ because $\left(K_{t}-1\right)=2$ for the infinite plate. Therefore $K_{e}$ can be understood as an approximation of the ratio of the average stress over length $\alpha$ and the nominal stress. The error of approximation is explicitly given for the problem of the circular hole in an infinite plate. The relative error The percent relative error defined by

$$
e_{r}=100 \frac{\sigma_{x}^{(d)}-\left(q\left(K_{t}-1\right)+1\right) \sigma_{\infty}}{\sigma_{x}^{(d)}} \quad \text { where } \quad q=\frac{1}{1+d / a}
$$

is shown in Fig. 3. The maximum relative error is $19.08 \%$ which occurs at $d / a=1.39$. In other words, if the fatigue limit depends on the average normal stress over the length $d$ and Peterson's formula would be used to estimate this average stress then the error could be as large as 19.08 percent for the circular hole in the infinite plate.

\section{VALIDATION}

The formulation and testing of mathematical models for the prediction of damage accumulation due to fatigue under standard conditions, i.e., periodic loading characterized by fixed amplitude and mean value, involves the following processes:

1. Conceptualization: (a) formulation of a mathematical model that establishes a relationship between certain functionals of the stress or strain field and failure initiation or crack propagation events, (b) virtual experimentation and (c) calibration. The end product of conceptualization is a mathematical model.

2. Validation: Experiments performed to test the predictive capabilities of a mathematical model. The quality of predictions is evaluated with reference metrics and criteria formulated prior to the validation experiments. In the case of models formulated for the prediction of damage accumulation caused by metal fatigue, the metric is the number of cycles to failure. The formulation 


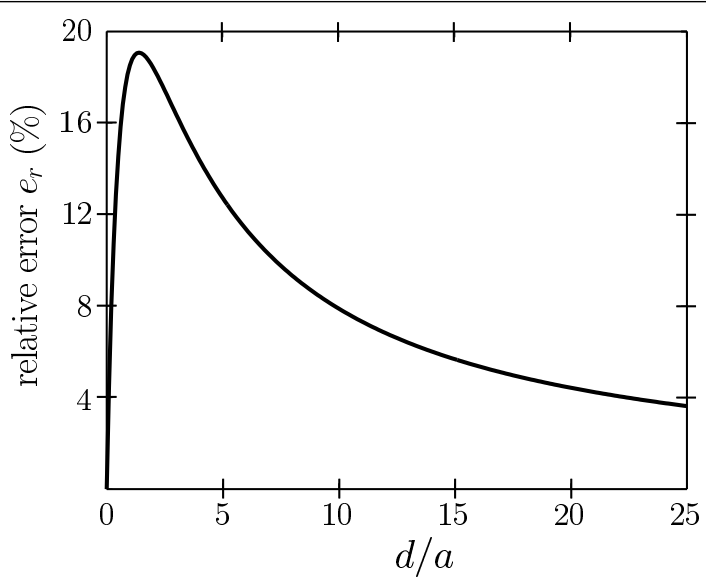

Figure 3. The relative error in Peterson's formula applied to the circular hole in an infinite plate.

of criteria is complicated by the fact that accumulation of fatigue damage is inherently stochastic.

Examples of conceptualization were given in Section 2 and a general framework for conceptualization is proposed in Section 5. It is now becoming standard practice to perform validation experiments. Many papers and case studies have been published on this subject see, for example, [15] - [20] and the references cited therein.

In principle, a model is rejected if it fails to meet established criteria. In practice it is generally not possible to assign pass/fail scores to mathematical models on the basis of the outcome of a limited number of experiments because statistical variability in material properties, loading, constraints and geometric attributes complicates comparisons of predicted and observed data. Nevertheless, it is possible to formulate a framework for quantifying a "degree of belief" in a mathematical model and rank alternative models on the basis of accumulated experience with the accuracy of predictions based on the models.

Two types of uncertainty are associated with damage accumulation models: Epistemic (cognitive) uncertainty and aleatory (statistical) uncertainty. The goal is of validation is to minimize epistemic uncertainties through objective evaluation and ranking of alternative models of damage accumulation.

\section{Fatigue tests of miCro-Machined AF1410 Steel specimens}

In the course of investigation of the effects of small surface defects on the fatigue life of aircraft components made of AF1410 steel one group of investigators recommended fitting truncated ellipsoids to the surface features and using eq. (2.11):

$$
K_{e}=q\left(K_{t}-1\right)+1
$$


with $q$ defined by eq. 2.2 and the notch radius $\varrho$ determined from the truncated ellipsoid. The truncated ellipsoid is characterized by three parameters; length, width and depth. It was called "pit metric" to convey the assumption that all surface features can be replaced for the purpose of fatigue life prediction with truncated ellipsoids. No limit was placed on the size of the pit metric, even though the notch radius in Peterson's model has an implied lower bound, as noted in Section 2. The implied assumption is that Peterson's model is capable of predicting crack nucleation events.

In order to test this assumption, twelve dog-bone specimens were machined from AF1410 steel. For each specimen the thickness of the test section was $3.05 \mathrm{~mm}(0.120$ in), the width was $25.4 \mathrm{~mm}$ (1.0 in). The test sections were hand-polished and six features were micro-machined into the test section of nine specimens: Two ellipsoidal features, two conical features, and two pill-shaped features. The features were located at 60 -degree intervals on the perimeter of a $12.7 \mathrm{~mm}(0.5 \mathrm{in})$ diameter circle, as shown in Fig. 4(a). One of the ellipsoidal and one of the pill-shaped features were oriented parallel with the direction of loading, the others were oriented perpendicular to it. The features were sufficiently far apart so that interaction among the features can be neglected. The remaining three specimens were tested without surface features.

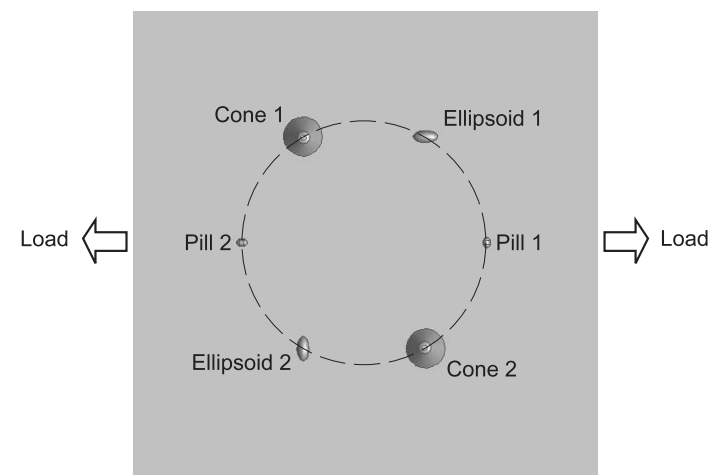

(a)

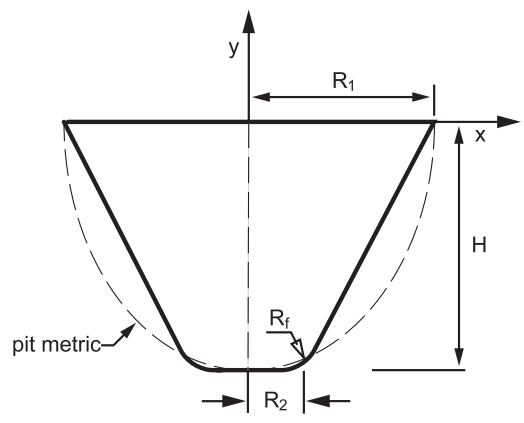

(b)

Figure 4. (a) Micro-machined features. The features lie on the perimeter of a $12.7 \mathrm{~mm}$ (0.5 in) diameter circle. (b) Conical feature. Relationship between the ellipsoidal pit metric and the micromachined cone.

An example of replacement of a conical feature, characterized by four parameters $\left(R_{1}=1.02 \mathrm{~mm}, R_{2}=0.33 \mathrm{~mm}, H=0.89 \mathrm{~mm}, R_{f}=0.39 \mathrm{~mm}\right)$, with a pit metric which in this case is characterized by only two parameters $\left(R_{1}, H\right)$ is shown in Fig. 4(b). The radius of curvature of the pit metric in the point $(0,-H, 0)$ is $\varrho_{\mathrm{pm}}=R_{1}^{2} / H=1.17 \mathrm{~mm}$. The ellipsoids are characterized by two radii $R_{1}=0.64$ $\mathrm{mm}, R_{2}=0.32 \mathrm{~mm}$ and the depth $H=0.32 \mathrm{~mm}$. The pill-shaped features are comprised of a cylinder and two spherical caps of radius $R=0.18 \mathrm{~mm}$, depth $H=0.18$ $\mathrm{mm}$ and combined length $L=0.57 \mathrm{~mm}$. 
The maximum applied stress was $1380 \mathrm{MPa}(200 \mathrm{ksi})$ the minimum stress was 138 $\mathrm{MPa}(20 \mathrm{ksi})$. Therefore the ratio of minimum to maximum stress was $R=0.1$. The constant amplitude cyclic load was maintained at $R=0.1$ for 1000 cycles then in each marker band block groups of 400 cycles at $R=0.7$ stress was followed by 8 cycles at $R=0.1$ stress.

The computed values of $K_{e}$, based on the nominal dimensions of the micro-machined features, are shown in Table1. According to Peterson's model the first occurrence of crack nucleation is expected at Ellipsoid 2, the long axis of which is oriented perpendicular to the load direction.

Table 1. The values of $K_{e}$ for the three micro-machined features computed from Peterson's formula using $\alpha=0.064 \mathrm{~mm}$.

\begin{tabular}{|l|c|c|c|c|c|}
\hline \hline Feature & $\varrho(\mathrm{mm})$ & $\alpha / \varrho$ & $q$ & $K_{t}$ & $K_{e}$ \\
\hline Cone & 0.391 & 0.164 & 0.8593 & 2.477 & 2.269 \\
Ellipsoid 2 & 0.635 & 0.101 & 0.9084 & 2.476 & 2.341 \\
Pill 1 & 0.178 & 0.360 & 0.7355 & 2.492 & 2.097 \\
\hline \hline
\end{tabular}

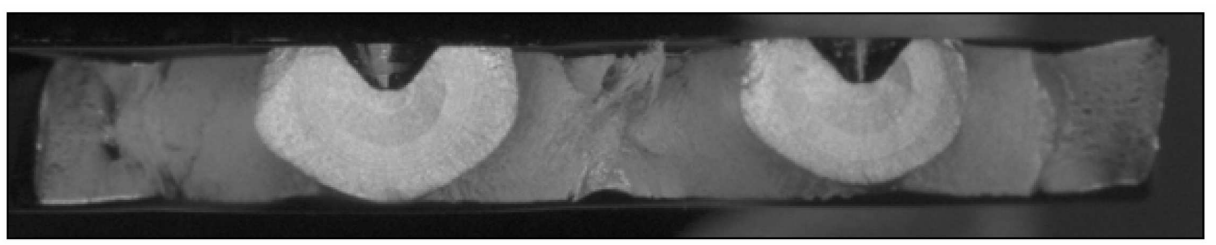

(a)

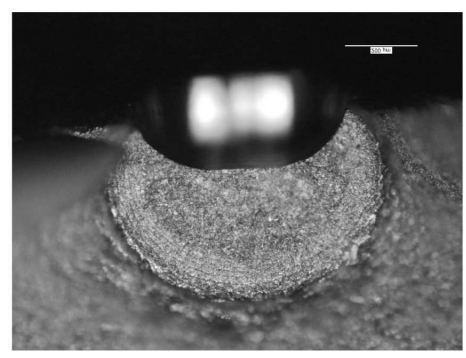

(b)

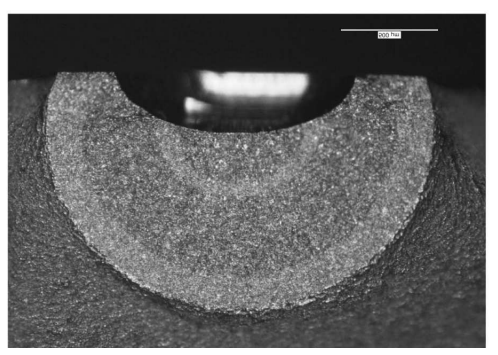

(c)

Figure 5. (a) Typical failure across conical features. (b) Secondary fatigue crack at a pill feature (specimen 598-2). (c) Secondary fatigue crack at an ellipsoidal feature (specimen 598-2).

Fractographic examination found that for all micro-machined specimens failure initiated at the conical features and the failure surface intersected the two conical 
features, a shown in Fig. 5(a). Secondary fatigue cracks developed at the ellipsoidal and pill-shaped features that did not grow to critical size prior to failure. Typical secondary cracks are shown in Fig. 5(b) and Fig. 5(c). The number of cycles to initiation, which is defined as the first occurrence of a $0.254 \mathrm{~mm}(0.01 \mathrm{in})$ crack, is shown in Table 2 .

Table 2. Results of fractographic examination of micromachined AF1410 dog-bone specimens. Number of $R=0.1$ cycles to crack initiation and total number of cycles. Maximum applied stress: 1379 $\operatorname{MPa}(200 \mathrm{ksi})$.

\begin{tabular}{|l|c|c|c|c|c|}
\hline \hline \multirow{2}{*}{ Specimen } & \multirow{2}{*}{ Surface } & \multicolumn{2}{|c|}{ primary conic } & \multicolumn{2}{c|}{ secondary conic } \\
\cline { 3 - 6 } & & $R=0.1$ & Total & $R=0.1$ & Total \\
\hline $598-4$ & smooth & 25,467 & 83,467 & - & - \\
$598-11$ & smooth & 24,212 & 80,212 & - & - \\
$598-14$ & smooth & 14,974 & 49,374 & - & - \\
\hline $598-1$ & notched & 1,767 & 6,567 & 1,800 & 6,600 \\
$598-2$ & notched & 1,700 & 3,300 & 1,822 & 5,822 \\
$598-3$ & notched & 1,968 & 4,368 & 1,971 & 4,371 \\
$598-5$ & notched & 1,521 & 5,760 & 1,934 & 6,173 \\
$598-8$ & notched & 1,418 & 4,209 & 1,869 & 4,660 \\
$598-9$ & notched & 1,828 & 5,700 & 1,936 & 5,808 \\
$598-10$ & notched & 1,500 & 3,100 & 1,833 & 3,433 \\
$598-12$ & notched & 1,483 & 4,633 & 1,550 & 4,700 \\
$598-13$ & notched & 1,790 & 6,502 & 1,970 & 6,682 \\
\hline Average & 1,664 & 4,904 & 1,854 & 5,361 \\
Standard deviation & 179 & 1,218 & 124 & 1,053 \\
\hline \hline
\end{tabular}

We note that the predictions were based on the nominal dimensions of the surface features. The actual dimensions were measured for only one of the specimens by means of white light interferometry (WLI). The largest deviations were found in $R_{f}=0.33$ $\mathrm{mm}(-15 \%)$ for Cone 2 and $L=0.69 \mathrm{~mm}(+17 \%)$ for Pill 1 . Such deviations notwithstanding, the results indicate a strong probability that Peterson's model is not capable of predicting crack initiation events for the conical feature. The reason for this is that the stress distribution in the vicinity of the cones is qualitatively different from the stress distribution in the vicinity of notches considered by Peterson and Neuber. Arguably this was not a fair test of Peterson's model because the assumptions incorporated in that model were not satisfied by the test article. This could have been determined through virtual experimentation prior to performing the physical experiments.

We have seen in Section 2 that Peterson's effective stress concentration factor $K_{e}$ can be understood as an approximation to the average stress over a materialdependent distance $\alpha$, which in two dimensions is equivalent to an area. The original intent in the development of $K_{e}$ was to estimate the fatigue life of machine elements 
with notches that are characterized by a dominant curvature, the radius of curvature being larger than approximately $\alpha / 4$. In those cases $K_{e}$ gives a reasonably good approximation to the average stress. The underlying idea of using average stress over a line, area or volume has no such restrictions however and can be implemented into finite element analysis programs without difficulty.

The stress distribution in the vicinity of (a) the conical feature and (b) representation of the conical feature by the pit metric is shown in Fig. 6. It is seen that the stress distribution at the conical feature is not related to a clearly defined curvature but the stress distribution at the pit metric is. The same holds for the other surface features as well. This indicates that the idea of replacing surface features with a pit metric has to be rejected.

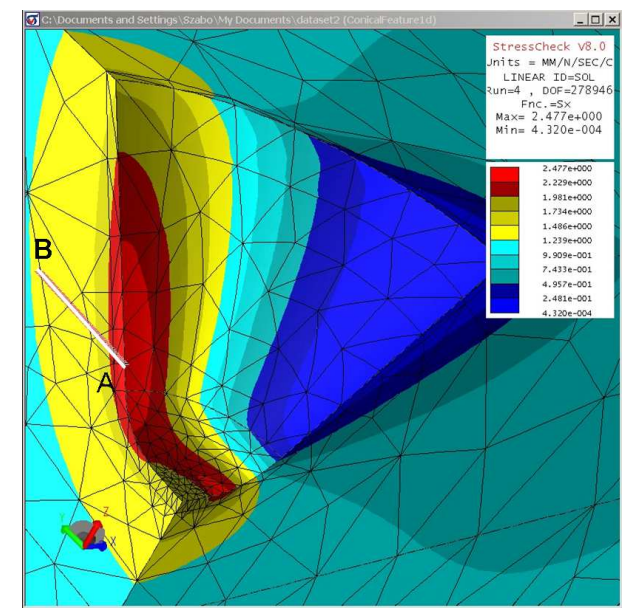

(a)

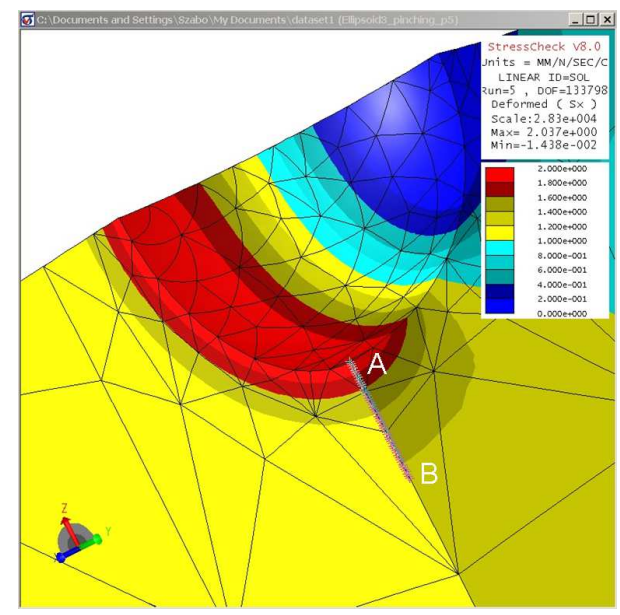

(b)

Figure 6. Stress distribution in the vicinity of (a) the conical feature and (b) representation of the conical feature by the pit metric. The nominal stress is $1 \mathrm{MPa}$ applied in the $\mathrm{x}$ - direction.

The values of $\sigma_{x}^{(d)} / \sigma_{\infty}$ for the three micro-machined features for various values of the averaging interval $d$ are shown in Table 3 . The averaging intervals were chosen by inspection so as to approximately maximize the average values. Examples of averaging intervals are shown by the lines labelad AB in Fig. 6.

It is seen that for the averaging interval ranging between $0.05 \mathrm{~mm}$ and $0.5 \mathrm{~mm}$ $\sigma_{x}^{(d)} / \sigma_{\infty}$ is largest for the conical features. Therefore a failure initiation model based on $\sigma_{x}^{(d)}$ would have correctly predicted that failure would begin at the conical features. Furthermore it would have predicted that the ellipsoid would be the next site of failure initiation, and the pill-shaped features would be last for any choice of $d$. This sequence of events was confirmed by fractographic examination. It is therefore possible to 
Table 3. The values of $\sigma_{x}^{(d)} / \sigma_{\infty}$ for the three micro-machined features.

\begin{tabular}{|l|c|c|c|c|}
\hline \hline Feature & $0.5 \mathrm{~mm}$ & $0.25 \mathrm{~mm}$ & $0.10 \mathrm{~mm}$ & $0.05 \mathrm{~mm}$ \\
\hline Cone & 1.640 & 1.894 & 2.165 & 2.296 \\
Ellipsoid 2 & 1.334 & 1.565 & 1.913 & 2.128 \\
Pill 1 & 1.189 & 1.352 & 1.683 & 1.953 \\
\hline \hline
\end{tabular}

conclude that predictions based on $\sigma_{x}^{(d)}$ are consistent with the results of experiments. The model based on the theory of critical distances passed the validation test.

\section{Generalization of Classical models for damage aCcumulation}

Examples of conceptualization were given in Sections 2 The conceptualization attributed to Neuber and the theory of critical distances are based on the assumption that a material constant, which has the dimension of length, exists and accumulation of fatigue damage can be correlated with the average stress over a line, area or volume characterized by that constant. This is contradicted by the experimental observation that the endurance limit depends on the size of the specimens, therefore the distance $d$ cannot be a material constant. Consequently there appear to be fundamental problems with the classical models described in Section 2

The classical models of driving force for damage accumulation exemplified by the work of Neuber, Peterson, Buch and others were developed for the estimation of the endurance limit of notched machine elements in tension, bending and shear. The computational tools available at that time were limited. The geometric stress concentration factors were determined mainly from classical solutions of the Navier-Lamé equations and photoelastic studies. The nominal stress was computed from simple formulae for bars, beams and shafts. Those limitations on computational tools no longer exist and there is a need for generalization of the classical models of damage accumulation to complicated parts, such as rotorcraft components, and small defects, such as those caused by manufacturing processes, corrosion, impact and wear. In this section the conceptual formulation of driving forces for damage accumulation is outlined. For the sake of simplicity in presentation the domain is assumed to be two-dimensional, unless otherwise noted, but the concept is not restricted to two dimensions.

Consider the neighborhood of a sharp or blunt notch, called stress riser. It is assumed in the following that the principles of continuum mechanics remain valid everywhere within the body up to the failure initiation event. At the site of damage accumulation the continuum model is likely to indicate strongly nonlinear behavior, such as the formation of shear bands, large strain and large rotation. This is the process zone, schematically indicated by the hatched area bounded by $\Gamma_{P Z}$ and $\Gamma$ in Fig. 7. 


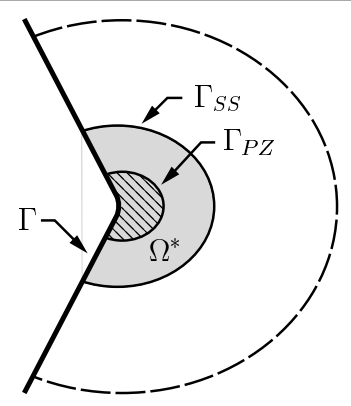

Figure 7. Notation.

Let $\mathbf{u}_{P Z}(\mathbf{x}, t)=\left\{u_{x}(\mathbf{x}, t) u_{y}(\mathbf{x}, t)\right\}_{P Z}$ be the solution of the general nonlinear continuum mechanics problem that accounts for strongly nonlinear behavior as well as heterogeneous material properties and let $\mathbf{u}_{S S}(\mathbf{x}, t)=\left\{u_{x}(\mathbf{x}, t) u_{y}(\mathbf{x}, t)\right\}_{P Z}$ be the solution of the continuum mechanics problem that accounts only for infinitesimal strain, small deformation and homogeneous material properties. Both $\mathbf{u}_{P Z}$ and $\mathbf{u}_{S S}$ are defined on the entire domain $\Omega$ and may be functions of time $t$. It is assumed that there is a subdomain $\Omega^{\star}$, bounded by $\Gamma_{S S}$ and $\Gamma$, such that outside of $\Omega^{\star} \mathbf{u}_{P Z} \approx \mathbf{u}_{S S}$. Specifically:

$$
\left\|\mathbf{u}_{P Z}(\mathbf{x}, t)-\mathbf{u}_{S S}(\mathbf{x}, t)\right\|_{\max } \leq \tau\left\|\mathbf{u}_{P Z}(\mathbf{x}, t)\right\|_{\max } \quad \mathbf{x} \in\left(\Omega-\Omega^{*}\right)
$$

where $\tau$ is some small tolerance. On the domain outside of $\Omega^{*}$ the usual infinitesimal strain and small deformation assumptions of continuum mechanics are reasonable representations of physical reality.

Implied in this assumption is that failure initiation, which depends on the solution of the highly nonlinear problem inside the process zone, is related to the solution of a linear or nonlinear problem of continuum mechanics for which the assumptions of infinitesimal strain, small deformation hold, even though these assumptions are violated inside the process zone. Consequently it should be possible to predict failure initiation events on the basis of $\mathbf{u}_{S S}$. An important special case is when $\mathbf{u}_{S S}$ can be well approximated by models based on linear elasticity. In such cases $\mathbf{u}_{S S}(\mathbf{x}, t)$ can be written as the product of a function of $\mathbf{x}$ and a function of $t$.

The key problem is identification of the driving force for damage accumulation, given $\mathbf{u}_{S S}(\mathbf{x}, t)$. Infinitely many conceptualizations are possible. For example, the following defines a family of possible definitions of driving force in terms of some functional $F\left(\mathbf{u}_{S S}\right)>0$ and a parameter $\alpha$ :

$$
G(F, \alpha, t, T)=\int_{\Omega_{\alpha}} F\left(\mathbf{u}_{S S}(\mathbf{x}, t), T\right) d V, \quad \mathbf{x} \in \mathbb{R}^{3}
$$

where $T$ represents temperature. The domain of integration $\Omega_{\alpha}$ depends on the magnitude of $F$ :

$$
\Omega_{\alpha}=\left\{\mathbf{x} \mid \alpha \leq F / F_{\lim }, 0<\alpha<1\right\}
$$


Various definitions of $F_{\text {lim }}>0$ are possible. The value of $F_{\text {lim }}$ may depend on the yield stress, yield strain, the ultimate tensile stress or some other value. The choice $\Omega_{\alpha}$ as a function of $F$ is related to the experimentally observed fact that the larger the volume subjected to elevated stress or strain, the greater the likelihood of failure.

Remark 3. In general, the solution $\mathbf{u}_{S S}$ is not known, only an approximation to $\mathbf{u}_{S S}$, which will be denoted by $\mathbf{u}_{F E}$, is known. Replacement of $\mathbf{u}_{S S}$ by $\mathbf{u}_{F E}$ is permissible only when $\left\|\mathbf{u}_{S S}-\mathbf{u}_{F E}\right\|_{\max }$ is sufficiently small on $\Omega-\Omega^{\star}$. This follows from the inequality:

$$
\left\|\mathbf{u}_{P Z}-\mathbf{u}_{S S}\right\|_{\max } \leq\left\|\mathbf{u}_{P Z}-\mathbf{u}_{F E}\right\|_{\max }+\left\|\mathbf{u}_{S S}-\mathbf{u}_{F E}\right\|_{\max } .
$$

Numerical accuracy is essential because unless the accuracy of the computed data is known it is not meaningful to compare experimental observations with predictions based on a mathematical model. This is because it would not be possible to tell whether the mathematical model is wrong or the numerical errors are too large, or both. In some cases a large error in the mathematical model is nearly canceled by a similarly large numerical error, leading to false conclusions [21].

Example 3. The region of integration $\Omega_{\alpha}$ is illustrated for the conical feature when $F=\sigma_{1}$, is defined as the first principal stress, $F_{\text {lim }}=\sigma_{\text {yld }}=1517 \mathrm{MPa}$ is defined as the yield stress of AF1410 steel and $\alpha=0.95$ in Fig. 8 .

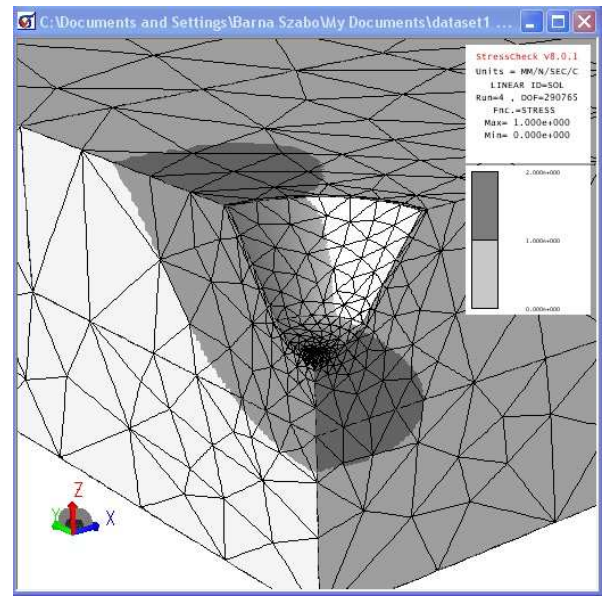

Figure 8. Example 3. The region of integration $\Omega_{\alpha}$ is highlighted for $\alpha=0.95$. Specifically, $\sigma_{1} \geq 0.95 \sigma_{\text {yld }}$ over the dark grey region.

\section{Closing Remarks}

Damage tolerance and flaw tolerance methods employed in the management of mechanical and structural systems have a common conceptual basis: The highly nonlinear processes of crack nucleation and crack propagation are typically controlled by stress and strain fields that can be determined to a high degree of accuracy from 
the solutions of mathematical models based on small displacement and infinitesimal strain theory.

Development of a predictive capability for crack nucleation and crack propagation based on small displacement and infinitesimal strain theory involves the definition of a driving force $G(F, \alpha, t, T)$, see eq. $\sqrt{5.2}$, and interpretation of the outcome of calibration experiments designed for determination of the parameters in $G$. The definition of $G$ involves expert opinion, i.e., subjective judgment. Nevertheless it is possible to rank alternative definitions objectively through the application of verification and validation procedures. For example, the results of fatigue tests of the micro-machined AF1410 steel specimens presented in Section 4 clearly indicate that $G$ defined as average stress over lengths ranging between 50 to $200 \mu \mathrm{m}$ is preferable to Peterson's definition of the driving force.

Neuber's seminal work on the investigation of the fatigue limit of notched machine elements should be understood as an attempt to define a particular driving force $G$. Specifically, Neuber's driving force was the average tensile or shear stress over a length which he assumed to be a material property. This length was calibrated for various metals and was shown to provide useful predictions for typical notch configurations in machine elements. The notch radii in the calibration experiments were greater than about $0.5 \mathrm{~mm}$. Scatter in the measured data tend to increase with decreasing notch radius. Furthermore, as seen in Section 4 , not all surface features can be characterized by a single notch radius. For example, the stress distribution in the vicinity of the conical feature is not associated with a notch radius (see Fig. 6). Therefore Neuber's and Peterson's estimate of $K_{e}$ is not applicable to the conical feature. Another difficulty is that the classical models do not account for the influence of size on fatigue limit.

There are conceptual problems also with mathematical models based on linear elastic fracture mechanics for the estimation of crack propagation rates. These problems arise because coupon tests designed for the determination of material parameters that characterize crack propagation are performed on test articles which do not meet the conditions of planar elasticity: The stress field at the intersection of the crack front with the surface of the test article is not the stress field assumed in LEFM and, furthermore, the crack front is typically curved. These conditions are typically ignored in the interpretation of calibration experiments, resulting in systematic errors when the results of calibration are applied to general crack configurations.

The class of driving fores defined in equations $(5.2)$ and $(5.3)$ do not have such limitations. Given the framework of verification and validation, it is possible to reinterpret the results of fatigue and fracture experiments with the objective to identify specific driving forces that have the best predictive capabilities, independent of notch configuration and size. This will minimize systematic errors in the prediction of crack nucleation and crack propagation events. 


\section{ACKNOWLEDGMENT}

This paper is an elaboration on the author's presentation at the MITACS Workshop entitled "Methodology of Validation and Verification" held in Banff, Alberta, Canada between April 28 and May 1, 2008. The author is indebted to the participants for lively discussions on V\&V issues. Special thanks are due to Professor Ivo Babuška, Dr. Paul Hoffman, Mr. David Rusk and Dr. Ricardo Actis.

\section{REFERENCES}

1. APPLIED VEHICLE TECHNOLOGY PANEL, TASK GROUP AVT-125, RESEARCH AND TECHNOLOGY ORGANISATION (NATO): Future Airframe Structural Lifing: Methods, Applications and Management. Report No. RTO-TR-AVT-125, Neuilly-surSeine Cedex, France, 2008.

2. MARIANO, P. M.: Multifield Theories in Mechanics of Solids. In Advances in Applied Mechanics 38, Edited by E. van der Giessen and T. Y. Wu, Academic Press, 2001, 2-95.

3. PETERSON, R. E.: Stress Concentration Factors. John Wiley \& Sons, New York, 1974.

4. PILKEY, W. D.: Peterson's Stress Concentration Factors. 2nd ed. John Wiley \& Sons, New York, 1997.

5. NEUBER, H.: Kerbspannungslehre. Springer-Verlag, Berlin, 1937.

6. KUHN, P. and HARDRATH, H. F., An Engineering Method for Estimating Notch-Size Effect in Fatigue Tests of Steel. NACA Technical Note 2805, 1952.

7. VIGILANTE, J. N., UNDERWOOD, J. H. and CRAYON, D.: Use of instrumented bolt and constant displacement bolt-loaded specimen to measure in-situ hydrogen crack growth in high-strength steels. Fatigue and Fracture Mechanics: 30th Volume, ASTM STP 1360. P. C. Paris and K. L. Jerina, Eds. American Society for Testing and Materials. West Conshohocken, PA, 2000, 377-387.

8. BUCH, A.: Notch-Size Effect of Steel Specimens - Verification of Some Calculation Methods. Z. Werkstofftech, 15, 1984, 338-348.

9. BABUŠKA, I. and SZABÓ, B.: On the generalized plane strain problem in thermoelasticity. Comput. Methods Appl. Mech. Engng. 195, 2006, 5390-5402.

10. TAYLOR, D.: The Theory of Critical Distances. A New Perspective in Fracture Mechanics. Elsevier, Oxford (2007).

11. TAYLOR, D.: The theory of critical distances. Engineering Fracture Mechanics. 7, 2008, $1696-1705$.

12. SUSMEL, L. and TAYLOR, D.: A novel formulation of the Theory of Critical Distances to estimate lifetime of notched components in the medium-cycle fatigue regime. Fatigue Fract. Engng. Mater. Struct. 30, 2007, 567-581.

13. SUSMEL, L.: The theory of critical distances: a review of its applications to fatigue. Engineering Fracture Mechanics. 7 2008, 1706-1724.

14. MUSKHELISHVILI, N. I.: Some Basic Problems of the Mathematical Theory of Elasticity, published in Russian in 1933, English translation of the 3rd edition: Groningen, Holland, 1953.

15. SZABÓ, B. A. and ACTIS, R. L.: On the role of hierarchic spaces and models in verification and validation. Comput. Methods Appl. Mech. Engrg. 198, 2009, 1273-1280. 
16. ROACHE, P. J.: Verification and Validation in Computational Science and Engineering Hermosa Publishers, Albuquerque, New Mexico, 1998.

17. ROACHE P. J.: Verification of Codes and Calculations. AIAA Journal 36, 1998, 696702 .

18. OBERKAMPF, W. L.: What are validation experiments? Experimental Techniques May/June, 2001, 35-40.

19. ASME V\&V 10-2006: Guide for Verification and Validation in Computational Solid Mechanics. The American Society of Mechanical Engineers, ISBN No. 0-7918-3042-X, 2006.

20. BABUŠKA, I., NOBILE, F. and TEMPONE, R.: A systematic approach to model validation based on Bayesian updates and prediction related rejection criteria. Comput. Methods Appl. Mech. Engrg. 197, 2008, 2517-2539.

21. SZABÓ, B. and BABUŠKA, I.: Finite Element Analysis. John Wiley \& Sons. Inc. New York, 1991. 